\title{
Uniportal Video-Assisted Thoracoscopic Surgery and Outcomes for Recurrent Primary Spontaneous Pneumothorax: Single-Institution Experience
}

\author{
Iskander Al-Githmi \\ Department of Surgery, Division of Cardiothoracic Surgery, King Abdulaziz University Hospital, Jeddah, Saudi Arabia \\ Email: algithmi@hotmail.com
}

How to cite this paper: Al-Githmi, I. (2018) Uniportal Video-Assisted Thoracoscopic Surgery and Outcomes for Recurrent Primary Spontaneous Pneumothorax: Single-Institution Experience. Surgical Science, 9, 122-127.

https://doi.org/10.4236/ss.2018.93013

Received: March 5, 2018

Accepted: March 23, 2018

Published: March 26, 2018

Copyright $\odot 2018$ by author and Scientific Research Publishing Inc. This work is licensed under the Creative Commons Attribution International License (CC BY 4.0).

http://creativecommons.org/licenses/by/4.0/

c) (i) Open Access

\begin{abstract}
Introduction: Primary spontaneous pneumothorax is relatively common condition in young adults. Uniportal video-assisted thoracoscopic surgery (uniportal VATS) has been accepted as a less invasive technique for the treatment of primary spontaneous pneumothorax. Strong evidence suggests that Uniportal VATS procedures are technically feasible and safe with excellent outcomes comparable to conventional VATS approach. Objectives: This article aims to discuss our experience with uniportal thoracoscopic approach as a valuable option in patients with recurrent spontaneous pneumothorax. Study Design: A retrospective study analysis between January 2014 and December 2016. Materials and Methods: From January 2014 to December 2016, 22 consecutive patients with unilateral recurrent spontaneous pneumothorax were to undergo uniportal video-assisted thoracic surgery (uniportal VATS). Their chronic residual postoperative pain, hospital stay and recurrence rate were analyzed. Results: Twenty-two patients with unilateral recurrent spontaneous pneumothorax were included; all received uniportal video-assisted thoracic surgery (uniportal VATS) and mechanical pleurodesis. Conclusions: We conclude that uniportal video-assisted thoracic surgery (uniportal VATS) demonstrated benefits to patients with primary spontaneous pneumothorax a safe, effective and also faster recovery, and decreased postoperative pain and short hospital stay.
\end{abstract}

\section{Keywords}

Primary, Pneumothorax, Uniportal, Video-Assisted Thoracic Surgery 


\section{Introduction}

Primary spontaneous pneumothorax is relatively common condition in otherwise healthy young adults. The typical patient is young, thin, tall, cigarette smoker and has acute chest pain with various degrees of cough and shortness of breath. The American College of Chest physicians have published guidelines for the management of primary spontaneous pneumothorax (PSP) [1]. Their reports confirmed that, after the first recurrence, PSP should be treated surgically. Uniportal video-assisted thoracoscopic surgery (VATS) is growing and has been widely accepted as a less invasive technique for the treatment of pneumothorax and offers the advantage of minimizing the extent of the surgical trauma. In 2004, Rocco et al. published their first paper about the use of the uniportal video-assisted thoracic surgery (VATS) as a unique minimal access surgical technique designed to perform lung resections in patients with PSP [2]. The aim of this study is analyze our first experience of uniportal VATS in primary spontaneous pneumothorax.

\section{Materials and Methods}

A retrospective study performed at our institution from January 2014 and December 2016. This study was approved by the local ethics committee, which waived the need for written informed consent Twenty-two patients " 3 women, 19 men, mean age, 28.5 years; range, 16 - 57 years" with unilateral primary spontaneous pneumothorax underwent uniportal video-assisted thoracic surgery and mechanical pleurodesis $(\mathrm{n}=22)$. Patient chronic post-operative residual pain, hospital stays and recurrence rate were analyzed. Patient demographic data and outcomes are presented in (Table 1).

Table 1. Demographic clinical data and outcomes.

\begin{tabular}{ll}
\hline Variable & Total $(\mathbf{n}=22)$ \\
\hline Age mean (years) & 28.5 \\
Gender, no. (\%) & $19(86 \%)$ \\
Male & $3(14 \%)$ \\
Female & $14(63 \%)$ \\
Smoking = yes & 91.6 \\
Operative time mean (min.) & $15(68 \%)$ \\
Histopathology = bleb & 3.5 \\
Chest Tube mean (days) & 4.01 \\
Hospital Stay mean (days) & 0.7 \\
Residual pain mean (18 months) & $0 \%$ \\
Postoperative recurrence rate (\%) &
\end{tabular}




\subsection{Inclusion Criteria}

All twenty-two patients admitted with unilateral spontaneous pneumothorax recurrence were included in the study. Diagnosis of pneumothorax made on the chest-x ray.

\subsection{Surgical Technique}

All twenty-two patients received uniportal video-assisted thoracic surgery (VATS) which was performed under general anesthesia with double lumen endotracheal intubation. Patients were placed in a lateral decubitus position with the arm abduct to allow the maximum upward displacement of scapula. One inch length single access incision was made along the anterior axillary line in the fifth intercostal space. Neither rib spreading nor wound retractors were used. A 30 degree telescope with attached camera was placed through the posterior part of the wound. The whole lung particular the apical segment of the upper lobe and superior segments of the lower lobe were inspected for blebs/bullae. These were resected with Echelon 60 Endopath stapler; Ethicon Endosurgery Corp., Cincinnati, OH, USA. The parietal pleural were abraded with diathermy scratch pad, mounted on a long Roberts clamp, particular attentions were paid to ensure that mechanical pleurodesis is thoroughly performed at the apical and lateral regions of parietal pleural surface. After hemostasis was achieved and no air leak from staple line was seen, a single chest tube size 28 French was inserted and brought out through the posterior part of the wound and secured to chest wall and connected to underwater seal suction with negative pressure of 20 centimeter water. The chest tube removed when there is no air leak.

\subsection{Patient's Follow-Up}

All twenty-two patients were followed-up at 6 months interval in our outpatient clinic for duration of 18 months. At each visit a chest $\mathrm{x}$ ray was performed and patient post-operative residual pain score was measured by a visual analogic score ranging from 0 to 4 . Description of each score is provided in Table 2.

\section{Results}

Uniportal VATS apical wedge resection was successfully performed in all twenty-two patients. The mean operative time was 91.6 minutes "range, 60 - 150 mins".

Table 2. The visual analogue scale.

\begin{tabular}{ll}
\hline Score & Description \\
\hline 0 & No pain at rest or on movement \\
1 & Mild pain on movement but none at rest \\
2 & Mild pain at rest but moderate on movement \\
3 & Moderate pain at rest but severe on movement \\
4 & Severe pain at rest and on any movement \\
\hline
\end{tabular}


The mean intraoperative blood loss was 40 milliliter. We had no surgical-related mortality or morbidity. The chest tube duration was ranging from $3-7$ with mean of 3.5 days and the mean length of hospital stay was 4 days "range, 3 - 8 days". Chronic post-operative pain measured by visual analogic score was ranging from 0 to 4 with mean of 0.7 scales.

\section{Discussion}

In 2004 uniportal VATS technique was first described by Gaetano Rocco [2]. He initially reported on his use of the uniportal technique for wedge resections either for diagnosis of interstitial lung disease or for treatment of primary spontaneous pneumothoraxes. In 2011 Gonzalez Rivas from Coruña University hospital in Spain report his first experience of uniportal VATS major lung resection for non-small cell lung cancer [3]. VATS resection of bullae/blebs is the treatment of choice for primary spontaneous pneumothorax and highly recommended to prevent recurrence [4] [5]. Uniportal VATS did not decrease the risk of recurrence of PSP. But the key factors to achieve success in uniportal approach are the angled $\left(30^{\circ}\right)$ thoracoscope and roticulator staplers [6] [7] [8]. However, VATS is not without complications and recent observations have quantified associated residual and neurological sequelae [9] [10]. In our study, we have demonstrated no complication rates using a single-port technique and there was no post-operative recurrence. A comparison between the standard three-port VATS and uniportal VATS for spontaneous pneumothorax was performed relating the efficacy of the uniportal approach [11]. Currently, this technique has been accepted worldwide and is part of routine practice in the field of thoracic surgery. The main outcome advantage of uniportal VATS is decreased post-operative pain and paresthesia. The use of only a single incision means less access trauma and only one intercostal space. In 2005 Jutley et al. first reported data on post-operative pain after uniportal VATS [11]. Chen et al. reported their results comparing between 20 PSP patients submitted to three-port VATS and 10 patients treated by uniportal VATS. The uniportal group experienced a lower postoperative pain in the first 24 hours measured by the visual analogic scale ranging 0 to 10 "three-port $4.95 \pm$ 0.39 vs. single port $4.5 \pm 0.7, \mathrm{P}=0.03$ " and reported a higher patient satisfaction scale "graded from grade1: excellent satisfaction to grade 4: poor satisfaction" [12]. Tamura et al. retrospectively compared their results of 37 patients in total, 18 3-port VATS operations and 19 single incision VATS [13]. A visual analog scale was used to assess pain scores and was recorded on postoperative days 0,1 , 3, 7 and 14. Pain scores were significantly higher in patients who underwent standard 3-port VATS on postoperative days 0, 1 and 3 than those who underwent uniportal VATS. There was no difference in length of chest tube duration, duration of surgery or hospital stay. The uniportal group did also report higher satisfaction scores, although this was not statistically significant., In our study, we have demonstrated no complication rates using a single-port technique, We observed that all patients did benefit from surgery with no post-operative recur- 
rence, This suggests that the technique allows for good exposure and adequate resection of the affected areas of the lung. Several reports have demonstrated the chronic pain and paresthesia represent major issue in three-ports technique which does not the issue in uniportal technique [10] [14]. We have demonstrated $91 \%$ uniportal patients reported no residual chronic pain while the remaining complaining of paresthesia and numbness sensation during 18 months follow up. The limitation of our study is small sample size and retrospective observational analysis. I believe a prospective randomized controlled trial should be carried out.

\section{Conclusion}

Uniportal VATS technique is a safe, reliable method and effective for treating primary spontaneous pneumothorax. The main outcome in our study is to decrease residual chronic post-operative pain and paresthesia.

\section{References}

[1] Baumann, M.H., Strange, C., Heffner, J.E., et al. (2001) Management of Spontaneous Pneumothorax: An American College of Chest Physicians Delphi Consensus Statement. Chest, 119, 590-602. https://doi.org/10.1378/chest.119.2.590

[2] Rocco, G., Martin-Ucar, A. and Passera, E. (2004) Uniportal VATS Wedge Pulmonary Resections. The Annals of Thoracic Surgery, 77, 726-728.

[3] Gonzalez, D., Paradela, M., Garcia, J., et al. (2011) Single-Port Video Assisted Thoracoscopic Lobectomy. Interactive Cardio Vascular and Thoracic Surgery, 12 514-515. https://doi.org/10.1510/icvts.2010.256222

[4] de Vos, B., Hendriks, J., Van Schil, P., Van Hee, R. and Hendrickx, L. (2002) Long-Term Results after Video-Assisted Thoracic Surgery for Spontaneous Pneumothorax. Acta Chirurgica Belgica, 102, 439-444. https://doi.org/10.1080/00015458.2002.11679348

[5] Passlick, B., Born, C., Haussinger, K. and Thetter, O. (1998) Efficiency of Video-Assisted Thoracic Surgery for Primary and Secondary Spontaneous Pneumothorax. The Annals of Thoracic Surgery, 65 324-327. https://doi.org/10.1016/S0003-4975(97)01128-4

[6] Migliore, M. and Deodato, G. (2001) A Single-Trocar Technique for Minimally Invasive Surgery of the Chest. Surgical Endoscopy, 15, 899-901. https://doi.org/10.1007/s004640090033

[7] Migliore, M., Giuliano, R., Aziz, T., et al. (2002) Four-Step Local Anesthesia and Sedation for Thoracoscopic Diagnosis and Management of Pleural Diseases. Chest, 121, 2032-2035. https://doi.org/10.1378/chest.121.6.2032

[8] Migliore, M. (2003) Efficacy and Safety of Single-Trocar Technique for Minimally Invasive Surgery of the Chest in the Treatment of Noncomplex Pleural Disease. The Journal of Thoracic and Cardiovascular Surgery, 126, 1618-1623. https://doi.org/10.1016/S0022-5223(03)00592-0

[9] Sihoe, A.D., Au, S.S., Cheung, M.L., Chow, I.K., Chu, K.M., Law, C.Y., Wan, M. and Yim, A.P. (2004) Incidence of Chest Wall Paresthesia after Video-Assisted Thoracic Surgery for Primary Spontaneous Pneumothorax. European Journal of Cardio-Thoracic Surgery, 25, 1054-1058. https://doi.org/10.1016/j.ejcts.2004.02.018 
[10] Passlick, B., Born, C., Sienel, W. and Thetter, O. (2001) Incidence of Chronic Pain after Minimal-Invasive Surgery for Spontaneous Pneumothorax. European Journal of Cardio-Thoracic Surgery, 19, 355-358. https://doi.org/10.1016/S1010-7940(01)00568-1

[11] Jutley, R.S., Khalil, M.W. and Rocco, G. (2005) Uniportal vs Standard Three-Port VATS Technique for Spontaneous Pneumothorax: Comparison of Post-Operative Pain and Residual Paraesthesia. European Journal of Cardio-Thoracic Surgery, 28, 43-46. https://doi.org/10.1016/j.ejcts.2005.02.039

[12] Chen, P.R., Chen, C.K., Lin, Y.S., et al. (2011) Single-Incision Thoracoscopic Surgery for Primary Spontaneous Pneumothorax. Journal of Cardiothoracic Surgery, 6, 58. https://doi.org/10.1186/1749-8090-6-58

[13] Tamura, M., Shimizu, Y. and Hashizume, Y. (2013) Pain Following Thoracoscopic Surgery: Retrospective Analysis between Single-Incision and Three-Port Video-Assisted Thoracoscopic Surgery. Journal of Cardiothoracic Surgery, 8, 153. https://doi.org/10.1186/1749-8090-8-153

[14] Lang-Lazdunski, L., Chapuis, O., Bonnet, P.M., Pons, F. and Jancovici, R. (2003) Videothoracoscopic Bleb Excision and Pleural Abrasion for the Treatment of Primary Spontaneous Pneumothorax: Long-Term Results. The Annals of Thoracic Surgery, 75, 960-965. https://doi.org/10.1016/S0003-4975(02)04544-7 